\title{
Histopathological findings of extra-ileal manifestations at initial diagnosis of Crohn's disease-related ileitis
}

\author{
Ian S. Brown ${ }^{1,2,3}$ • Gregory Miller ${ }^{1,3}$ • Mark L. Bettington ${ }^{1,3,4} \cdot$ Christophe Rosty $^{1,3,5}$
}

Received: 10 April 2017 / Accepted: 12 April 2017 / Published online: 19 April 2017

(C) Springer-Verlag Berlin Heidelberg 2017

\section{Dear Editor,}

We thank Doctors Villanacci and Bassotti for their kind comments and their interest in our paper [1]. We agree with their point that the diagnosis of Crohn's disease (CD) involving the upper gastrointestinal tract requires knowledge that Crohn's disease-related inflammation also exists in the terminal ileum and/or colon. Since upper gastrointestinal tract endoscopy with biopsy may be the initial investigation for symptoms related to $\mathrm{CD}$, we believe that pathologists should be vigilant to the possibility that otherwise unexplained active chronic inflammation in the upper gastrointestinal tract could be a manifestation of CD, even without evident granuloma formation. Hence, CD should be included in the differential diagnosis of such cases and the clinician encouraged to look in the lower gastrointestinal tract for additional evidence of disease.

The preliminary finding that focal neutrophil infiltration of the surface of the ileal villi represents an early morphological marker of CD is very interesting and certainly warrants further investigation. The separation of CD-related ileitis from non $\mathrm{CD}$ ileitis is an ongoing clinical problem for us and others [2] and was a prime motivator for this study. Unfortunately to date, outside of the identification of granulomas, which are almost always due to CD in our practice, we have not been able to define any reliable means to separate $\mathrm{CD}$ from non CD-related ileitis by histopathology alone.

\section{References}

1. Brown IS, Miller GC, Bettington ML, Rosty C (2016) Histopathological findings of extraileal manifestations at initial diagnosis of Crohn's disease-related ileitis. Virchows Arch 469:515522

2. Courville EL, Siegel CA, Vay T, Wilcox AR, Suriawinata AA, Srivastava A (2009) Isolated asymptomatic ileitis does not progress to overt Crohn disease on long-term follow-up despite features of chronicity in ileal biopsies. Am J Surg Pathol 33:1341-1347
Ian S. Brown

IanBrown@envoi.com.au

Envoi Specialist Pathologists, Brisbane, QLD, Australia

2 Anatomical Pathology, Pathology Queensland, Royal Brisbane and Women's Hospital, Brisbane, QLD, Australia

3 School of Medicine, University of Queensland, Brisbane, QLD, Australia

4 The Conjoint Gastroenterology Laboratory, QIMR Berghofer Medical Research Institute, Brisbane, QLD, Australia

5 Department of Pathology, The University of Melbourne, Melbourne, VIC, Australia 